\title{
Pacific
}

Journal of

Mathematics

\section{REMARKS ON A KÜNNETH FORMULA FOR FOLIATED DE RHAM COHOMOLOGY}

MÉlANIE BERTELSON 


\title{
REMARKS ON A KÜNNETH FORMULA FOR FOLIATED DE RHAM COHOMOLOGY
}

\author{
MÉLANIE BERTELSON
}

\begin{abstract}
The validity of the Künneth formula for foliated cohomology, that is, for the tangential de Rham cohomology of a foliated manifold, is investigated. The main difficulty encountered is the non-Hausdorff nature of the foliated cohomology spaces, forbidding the completion of the tensor product. We present versions of the Künneth formula when both factors have Hausdorff foliated cohomology and when one factor has finite-dimensional foliated cohomology and a compact underlying manifold. We also give a counterexample to an alternative version of the Künneth formula. The proof of the second result involves a right inverse for the foliated de Rham differential.
\end{abstract}

\section{Introduction}

The tangential de Rham cohomology or foliated cohomology of a foliated manifold $(M, \mathscr{F})$ is the cohomology of the complex obtained by forming the quotient of the Fréchet space of ordinary smooth forms on the manifold by those who vanish along the leaves of the foliation. Our initial interest for this cohomology comes from the observation that its vanishing in degree two may, under certain circumstances, be an obstruction to existence of a foliated symplectic structure, or equivalently, a regular Poisson structure whose underlying foliation is $\mathscr{F}_{F}$ (see [Bertelson 2001]). Among the tools for computing de Rham cohomology is the Künneth formula which asserts that the cohomology space of a product is isomorphic to the completed tensor product of the cohomology spaces of the factors via the map

$$
\varphi: \bigoplus_{p+q=n} H^{p}(M) \otimes H^{q}(N) \rightarrow H^{n}(M \times N), \quad a \otimes b \mapsto a \wedge b .
$$

This map indeed induces a map on foliated cohomology but because these spaces do not generally enjoy the Hausdorff separation property, the completion of the tensor product may not even be defined.

The results obtained so far and shown in the present paper are:

MSC2000: primary 46A32, 46E10, 53C12, 53D17; secondary 46A63, 46M18, 46A04.

Keywords: foliation, tangential de Rham cohomology, Künneth, tensor product, right inverse. 
(1) The Künneth formula is valid when the foliated cohomology spaces of both factors are Hausdorff. This is a consequence of a result of Grothendieck, described in [Schwartz 1954]. We have nevertheless included a relatively detailed proof in Section 2.

(2) It is also valid when the foliated cohomology of one of the factors is finitedimensional and the underlying manifold of that same factor is compact. Notice that it is not necessary to complete the tensor product in that case. This result was already known when one of the factors is a one-leaf foliation [El Kacimi-Alaoui 1983; Moore and Schochet 2006]. Our proof requires the construction of a right inverse for the foliated de Rham differential. It is based on results in the theory of splitting of exact sequences of Fréchet spaces [Meise and Vogt 1997; Vogt 2004].

(3) In the simple case where one of the factors, say $(M, \mathscr{F})$, has a non-Hausdorff foliated cohomology and the other factor, say $(N, \mathscr{G})$, is a manifold foliated by its points, a natural alternative version to the Künneth formula would involve $C^{\infty}\left(N, H^{*}(\mathscr{F})\right)$ in place of the completed tensor product. Nevertheless, we have constructed on the torus $\mathbb{T}^{2}$ foliated by Liouville slope lines a smooth family of exact forms - representing thus the zero element in $C^{\infty}\left(N, H^{*}(\mathscr{F})\right)$ - which is not the coboundary of any continuous family of functions - corresponding therefore to a nonzero element in $H^{*}(\mathscr{F} \times \mathscr{G})$.

Many relevant questions remain unanswered:

- Does a more sophisticated version of the Künneth formula, involving some type of higher order functors, hold in a non-Hausdorff situation?

- Does the foliated de Rham differential still admit a right inverse when the assumption of compactness or finite-dimensionality of the foliated cohomology is relaxed?

Finally, the results of this paper may apply to other cohomologies. We have in mind the Poisson cohomology of a Poisson manifold (not surprisingly, the tangential Poisson cohomology of a regular Poisson structure is isomorphic to the foliated cohomology of the induced foliation). For instance, the Künneth formula for Poisson cohomology is valid when the cohomology spaces are Hausdorff.

\section{Preliminaries}

Let $(M, \mathscr{F})$ be a foliated manifold, that is, a smooth Hausdorff second countable manifold $M$ endowed with a smooth foliation $\mathscr{F}$. The space of smooth $p$-forms, $p \geq 0$, is denoted by $\Omega^{p}(M)$ (a smooth 0 -form is just a smooth function) and the space of all forms by $\Omega^{*}(M)$. The weak $C^{\infty}$ topology provides $\Omega^{p}(M)$ (and $\Omega^{*}(M)$ ) with the structure of a nuclear Fréchet space. We are interested in the 
nuclear property because it guarantees uniqueness of the completion of the tensor product with any other Fréchet space.

Recall that a Fréchet space is a locally convex, metrizable, complete topological vector space. We will not attempt to explain the nuclear property here, but rather refer to Sections 47 and 50 [Trèves 1967], henceforth, abbreviated as [T]. For our purpose it is sufficient to know that the set of smooth functions on an open subset of $\mathbb{R}^{n}$ is nuclear (Corollary of Theorem 51.5 in [T, p. 530]), that a product of nuclear spaces is nuclear and that a Hausdorff projective limit of nuclear spaces is nuclear (Proposition 50.1 in [T, p. 514]). Indeed, $\Omega^{p}(M)$ is the projective limit of the spaces $\Omega^{p}\left(\phi_{\alpha}\left(U_{\alpha}\right)\right)$, where $\left(U_{\alpha}, \phi_{\alpha}\right)$ runs through an atlas on $M$. We will occasionally write TVS for topological vector space.

Consider the space $\Omega^{p}(M, \mathscr{F})=\left\{\omega \in \Omega^{p}(M):\left.\omega\right|_{F}=0 \forall\right.$ leaf $\left.F\right\}$ of forms vanishing along the leaves of $\mathscr{F}$. It is a closed subspace of $\Omega^{p}(M)$. Thus the quotient $\Omega^{p}(M) / \Omega^{p}(M, \mathscr{F})$ is a Fréchet nuclear space as well (see [T, p. 85 and Proposition 50.1, p. 514]). It is the space of foliated p-forms. The de Rham differential $d: \Omega^{*}(M) \rightarrow \Omega^{*+1}(M)$ which is a continuous linear map induces the foliated de Rham differential $d_{\mathscr{F}}: \Omega^{*}(\mathscr{F}) \rightarrow \Omega^{*+1}(\mathscr{F})$ with like properties. The space of $d_{\mathscr{F}-}$

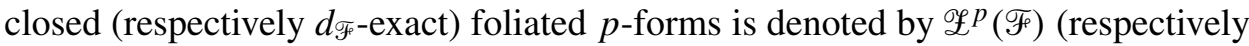
$\left.\mathscr{B}^{p}(\mathscr{F})\right)$. The cohomology $H^{*}(\mathscr{F})=\mathscr{L}^{*}(\mathscr{F}) / \mathscr{B}^{*}(\mathscr{F})$ is called the foliated (de Rham) cohomology of $(M, \mathscr{F})$.

Remark 1.1. The (ordinary) de Rham differential is always a homomorphism, that is, the image of an open subset of $\Omega^{p}(M)$ under $d$ consists of a relative open subset of $d\left(\Omega^{p}(M)\right)$. This is a consequence of the fact that a form is exact if and only if its integral over any closed cycle vanishes, showing that exact forms are a closed subset which by the open mapping theorem for metrizable and complete topological vector spaces implies that $d$ is open (Theorem 17.1 in [T, p. 170]). In contrast, the differential $d_{\mathscr{F}}$ need not be a homomorphism, as illustrated by Example 1.2 which describes the Liouville slopes foliations on the torus $\mathbb{T}^{2}$. Observe that assuming that $d_{\mathscr{F}}$ is open is equivalent to assuming that $\mathscr{B}^{*}(\mathscr{F})$ is closed (by the open mapping theorem for one direction and the observation that the image by a homomorphism of a complete metrizable TVS is a closed space for the other direction) or that the cohomology $H^{*}(\mathscr{F})$ is Hausdorff.

In this connection, the following examples are useful to keep in mind.

Example 1.2 (Kronecker foliations). Consider the foliation of $\mathbb{R}^{2}$ by parallel lines of slope $\alpha \in \mathbb{R}$. Invariant under the action of $\mathbb{Z}^{2}$ by translations, this foliation induces a foliation, denoted $\mathscr{F}_{\alpha}$, on the torus $\mathbb{T}^{2}$. The leaves are circles when $\alpha$ is a rational number and are dense lines otherwise. The foliated de Rham cohomology of $\mathscr{F}_{\alpha}$ for $\alpha$ irrational depends on the type of irrational number considered. More specifically, it is infinite-dimensional and non-Hausdorff (with a one-dimensional 
Hausdorff quotient) when $\alpha$ is a Liouville number (see Definition 1.3), and onedimensional and Hausdorff otherwise. The proof of this well-known fact can be found in [Haefliger 1980; Moore and Schochet 2006] and will appear implicitly in Section 4.

Definition 1.3. A Liouville number $\alpha$ is an irrational number that is well approximated by rational numbers. More precisely, for all integers $p \geq 1$, there exist relatively prime integers $m, n$ with $n>1$ such that

$$
\left|\alpha-\frac{m}{n}\right|<\frac{1}{|n|^{p}} \text {. }
$$

A typical example of such a number is Liouville's constant $\sum_{k=1}^{\infty} 10^{-k !}$. Liouville numbers are transcendental because an algebraic number $\alpha$ of degree $p \geq 2$ admits a constant $c$ such that

$$
\left|\alpha-\frac{m}{n}\right|>\frac{c}{|n|^{p}},
$$

for all integers $m, n$ with $n>0$. On the other hand $e$ and $\pi$, for instance, are not Liouville, as are uncountably many transcendental numbers. The set of Liouville numbers is a countable intersection of open dense sets and has measure zero. A non-Liouville number is sometimes called a generic number.

Example 1.4. Let $(M, \mathscr{F})$ be a foliation that has a vanishing $k$-cycle, that is, a smooth foliated map $v:\left(S^{k} \times[0,1], \mathscr{F}_{\pi}\right) \rightarrow(M, \mathscr{F})$, where $S^{k}$ is a sphere of dimension $k$ and $\mathscr{F}_{\pi}$ is the foliation by the fibers of the canonical projection $\pi: S^{k} \times[0,1] \rightarrow[0,1]$, such that the image of $S^{k} \times\{t\}$ is homotopically trivial in its leaf for each $t$ except $t=0$. A $p$-dimensional foliation from which a point is removed carries a vanishing $(p-1)$-cycle. We explain hereafter, in the specific case of a punctured foliation $(M, \mathscr{F})=\left(N-\{q\},\left.\mathscr{G}\right|_{N-\{q\}}\right)$, how the presence of the vanishing $(p-1)$-cycle implies that $H^{p}(\mathscr{F})$ is non-Hausdorff and infinite-dimensional. The argument can certainly be extended to a larger class of vanishing cycles.

Observe that our vanishing cycle can be "filled", in the sense that there exists a foliated map $\bar{v}:\left(D^{p} \times[0,1]-\operatorname{int} D^{p} \times\{0\}, \mathscr{F}_{\pi}\right) \rightarrow(M, \mathscr{F})$ that extends $v$. Let $\Omega$ be a foliated volume form on $(N, \mathscr{G})$ and let $f$ be a smooth function on $M$ approaching infinity near the puncture. Then $f \Omega$ is a foliated closed $p$-form on $M$ than cannot be foliated exact. Indeed, suppose on the contrary that $f \Omega=d_{\mathscr{F}} \alpha$. Then, by Stokes' theorem,

$$
\int_{\bar{v}\left(D^{p} \times\{t\}\right)} f \Omega=\int_{v\left(S^{p-1} \times\{t\}\right)} \alpha .
$$

Clearly, as $t$ approaches 0 , the right-hand side converges to $\int_{v\left(S^{p-1} \times\{0\}\right)} \alpha$ while the left-hand side diverges, yielding a contradiction. Besides, it is not too difficult to construct an example of a nonexact $p$-form of this type that is the limit of a 
sequence of exact forms, showing that the set of foliated exact forms is not closed in the set of foliated closed forms.

\section{Künneth formula when the cohomology is Hausdorff}

The main result of the present section, that is, a Künneth formula for foliated cohomology when the foliated cohomology of each factor is Hausdorff, is not original, as it is essentially a consequence of a theorem due to Grothendieck and described in [Schwartz 1954]. (A proof in terms of sheaves can also be found in the literature, namely in [Bredon 1997].) Nevertheless we give a relatively detailed explanation of the proof, with systematic references to the book [Trèves 1967] (referred to as $[\mathrm{T}]$ ) for the background functional analysis, believing that some readers might find it useful to have the proof expressed in a language familiar to differential geometers with references from just one very well-written book.

Let $(M, \mathscr{F})$ and $(N, \mathscr{G})$ be two foliated manifolds both having the property that the foliated de Rham differential is a homomorphism. Consider the (algebraic) tensor product $\Omega^{p}(\mathscr{F}) \otimes \Omega^{q}(\mathscr{G})$. There are two natural ways to construct a topology on the tensor product of two locally convex Hausdorff topological vector spaces, namely the $\varepsilon$ and the $\pi$ topology (Sections 42 and 43 in [T]), thus yielding two different completions of the tensor product. However, when one of the factors is Fréchet nuclear, both topologies coincide (Theorem 50.1 in [T, p. 511]). So in our case we can ignore this issue and write $\Omega^{p}(\mathscr{F}) \widehat{\otimes} \Omega^{q}(\mathscr{G})$ for the completionwith respect to this unique natural topology — of the tensor product of $\Omega^{p}\left(\mathscr{F}_{F}\right)$ with $\Omega^{q}(\mathscr{G})$. The tensor product of two continuous linear maps $f_{1}: E_{1} \rightarrow F_{1}$ and $f_{2}: E_{2} \rightarrow F_{2}$ between nuclear Fréchet spaces is a continuous linear map

$$
f_{1} \otimes f_{2}: E_{1} \otimes E_{2} \rightarrow F_{1} \otimes F_{2} \subset F_{1} \widehat{\otimes} F_{2},
$$

by Proposition 43.6 in [T, p. 439]; this induces a continuous linear map $f_{1} \widehat{\otimes} f_{2}$ : $E_{1} \widehat{\otimes} E_{2} \rightarrow F_{1} \widehat{\otimes} F_{2}$ between the completions. In general, the completion of a Hausdorff locally convex TVS $E$ is denoted by $\hat{E}$ and the extension of a continuous linear map $u: E \rightarrow F$ to the completions by $\hat{u}: \hat{E} \rightarrow \hat{F}$ [T, Theorem 5.1, p. 39].

Consider the tensor product complex $\left(\Omega^{*}(\mathscr{F}) \widehat{\otimes} \Omega^{*}(\mathscr{G}), d\right)$ defined as

$$
\left(\Omega^{*}(\mathscr{F}) \widehat{\otimes} \Omega^{*}(\varphi)\right)^{n} \stackrel{\text { def }}{=} \bigoplus_{p+q=n} \Omega^{p}(\mathscr{F}) \widehat{\otimes} \Omega^{q}(\mathscr{G}),
$$

with differential $d=d_{\mathscr{F}} \widehat{\otimes} 1+\varepsilon \widehat{\otimes} d \varphi$, where $\varepsilon(\omega)=(-1)^{p} \omega$ when $\omega$ is a foliated form of degree $p$. It follows from general considerations that $\Omega^{*}(\mathscr{F}) \widehat{\otimes} \Omega^{*}(\varphi)$ is a nuclear Fréchet space (Proposition 50.1 in [T, p. 514]) as well and that $d$ is a homomorphism. The latter assertion is a consequence of Proposition 43.9 in [T, p. 441] and the fact that the sum of two homomorphisms is a homomorphism. 
There is a natural map $\varphi$ between and $\Omega^{*}(\mathscr{F}) \widehat{\otimes} \Omega^{*}(\mathscr{G})$ and $\Omega^{*}(\mathscr{F} \times \mathscr{G})$, given by extension of the map

$$
\begin{aligned}
\underline{\varphi}: \Omega^{*}(\mathscr{F}) \otimes \Omega^{*}(\mathscr{G}) & \rightarrow \Omega^{*}(\mathscr{F} \times \mathscr{G}) \\
\sum_{i=1}^{I} \alpha_{i} \otimes \beta_{i} & \mapsto p_{M}{ }^{*}\left(\alpha_{i}\right) \wedge p_{N}{ }^{*}\left(\beta_{i}\right),
\end{aligned}
$$

where $p_{M}$ and $p_{N}$ denote the projections of $M \times N$ onto $M$ and $N$. It is clearly a cochain map $\left(\varphi \circ d=d_{\mathscr{F} \times} \mathscr{G} \circ \varphi\right)$, and so induces a map on foliated cohomology.

Theorem 2.1 (Künneth formula). The map $\varphi$ is an isomorphism on cohomology:

$$
H^{n}(\mathscr{F} \times \mathscr{G}) \cong\left(H^{*}(\mathscr{F}) \widehat{\otimes} H^{*}(\mathscr{G})\right)^{n} .
$$

This is a direct consequence of the following two results:

Theorem 2.2 (Grothendieck; see [Schwartz 1954]). Let $\left(E^{*}, d_{E}\right),\left(F^{*}, d_{F}\right)$ be two differential complexes of Fréchet spaces and homomorphisms. Suppose that the $E^{p}$ 's are nuclear. Consider the differential complex $\left(E^{*} \widehat{\otimes} F^{*}, d\right)$ with $d=$ $d_{E} \widehat{\otimes} 1+\varepsilon \widehat{\otimes} d_{F}$. Then $H^{*}(E \widehat{\otimes} F) \cong H^{*}(E) \widehat{\otimes} H^{*}(F)$.

Proposition 2.3. The differential complexes

$$
\left(\Omega^{*}(\mathscr{F}) \widehat{\otimes} \Omega^{*}(\mathscr{G}), d\right) \text { and }\left(\Omega^{*}(\mathscr{F} \times \mathscr{G}), d_{\mathscr{F} \times \mathscr{G}}\right)
$$

are isomorphic under the map $\varphi$.

The proof of Theorem 2.2 relies mostly on the next two lemmas:

Lemma 2.4 [Grothendieck 1955]. Let E, F, $G$ and $H$ be four Fréchet spaces with either $E, F$ and $G$ nuclear or $H$ nuclear. Let $u: E \rightarrow F$ and $v: F \rightarrow G$ be linear homomorphisms such that

$$
0 \rightarrow E \stackrel{u}{\rightarrow} F \stackrel{v}{\rightarrow} G \rightarrow 0
$$

is a short exact sequence. Then the sequence

$$
0 \rightarrow E \widehat{\otimes} H \stackrel{u \widehat{\otimes i} \mathrm{i}}{\longrightarrow} F \widehat{\otimes} H \stackrel{v \widehat{\otimes} \mathrm{id}}{\longrightarrow} G \widehat{\otimes} H \rightarrow 0
$$

is a short exact sequence of Fréchet spaces and linear homomorphisms as well.

Proof. That $u \widehat{\otimes}$ id is one-to-one and $v \widehat{\otimes}$ id is onto follows from Propositions 43.6 and 43.9, respectively, in [T, pp. 440-441]. Exactness at $F \widehat{\otimes} H$ is argued as follows. Firstly, observe that

$$
0 \rightarrow E \otimes H \stackrel{u \otimes \text { id }}{\longrightarrow} F \otimes H \stackrel{v \otimes \text { id }}{\longrightarrow} G \otimes H \rightarrow 0
$$

is a short exact sequence of homomorphisms. Indeed, the corollary of Proposition 43.7 in [T, p. 441] implies that $u \otimes$ id is a homomorphism. As for $v \otimes$ id, it suffices to 
know that a basis of neighborhoods of 0 for the $\pi$-topology consists of the convex hulls of sets of type $U \otimes V=\{u \otimes v: u \in U$ and $v \in V\}$, where $U$ (respectively $V$ ) is a balanced neighborhood of 0 in the first factor (respectively second factor). In other words, $U$ is a neighborhood of 0 such that $\lambda u \in U$, for all $|\lambda| \leq 1, u \in U$. Therefore, $G \otimes H \cong F \otimes H / u \otimes \operatorname{id}(E \otimes H)$. Secondly, it is not difficult to prove if $E$ is a metrizable TVS and if $N \subset E$ is a closed subspace then

$$
\widehat{E / N} \cong \hat{E} / \hat{N}
$$

where $\hat{N}$ denotes the closure of $N$ in the completion $\hat{E}$ of $E$.

Lemma 2.5. Let $\left(A^{*}, d_{A}\right),\left(B^{*}, d_{B}\right)$ and $\left(C^{*}, d_{C}\right)$ be three differential complexes of metrizable complete TVS's and homomorphisms and let

$$
0 \rightarrow A^{*} \stackrel{f}{\rightarrow} B^{*} \stackrel{g}{\rightarrow} C^{*} \rightarrow 0
$$

be a short exact sequence of differential complexes with $f, g$ continuous maps (hence homomorphisms by the open mapping theorem). Then the usual long exact sequence

$$
\cdots \rightarrow H^{*}(A) \stackrel{f_{*}}{\rightarrow} H^{*}(B) \stackrel{g_{*}}{\rightarrow} H^{*}(C) \stackrel{\nu}{\rightarrow} H^{*+1}(A) \rightarrow \cdots
$$

is well-defined with $f_{*}, g_{*}$ and $v$ homomorphisms.

Proof. Since $d_{A}, d_{B}$ and $d_{C}$ are homomorphisms, all spaces involved (that is, cocycles, coboundary and quotients of the formers by the latter) are complete metrizable spaces. The open mapping theorem implies that any surjective continuous linear map between those spaces will be a homomorphism. The only thing that requires a proof is therefore the continuity of $v$. This is easily seen by chasing open sets in the diagram providing the construction of $v$, namely,

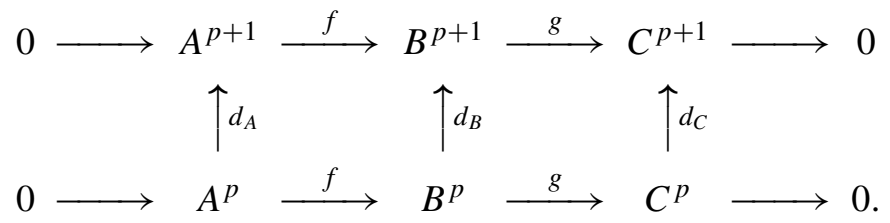

This completes the proof.

Proof of Theorem 2.2. We introduce some notation:

$$
\begin{aligned}
& \mathscr{L}^{p}=E^{p} \cap \operatorname{Ker} d_{E}, \quad \mathscr{B}^{p}=E^{p} \cap \operatorname{Im} d_{E}, \quad H^{p}=\mathscr{L}^{p} / \mathscr{B}^{p}, \\
& \mathscr{L}^{\prime p}=F^{p} \cap \operatorname{Ker} d_{F}, \quad \mathscr{R}^{\prime p}=F^{p} \cap \operatorname{Im} d_{F}, \quad H^{\prime p}=\mathscr{L}^{\prime p} / \mathscr{B}^{\prime p} .
\end{aligned}
$$

Now consider the following exact sequences of linear homomorphisms:

$$
\begin{array}{ll}
0 \rightarrow \mathscr{L}^{p} \rightarrow E^{p} \rightarrow \mathscr{B}^{p+1} \rightarrow 0, & 0 \rightarrow \mathscr{L}^{\prime p} \rightarrow F^{p} \rightarrow \mathscr{B}^{p+1} \rightarrow 0, \\
0 \rightarrow \mathscr{B}^{p} \rightarrow \mathscr{L}^{p} \rightarrow H^{p} \rightarrow 0, & 0 \rightarrow \mathscr{R}^{\prime p} \rightarrow \mathscr{L}^{\prime p} \rightarrow H^{\prime p} \rightarrow 0 .
\end{array}
$$


By Lemma 2.4, they induce the following other exact sequences of linear homomorphisms (obtained by tensoring with a fixed space and the identity map), where we have omitted the superscripts *:

$$
\begin{aligned}
& 0 \rightarrow(\mathscr{E} \widehat{\otimes} F)^{n} \rightarrow(E \widehat{\otimes} F)^{n} \rightarrow(\mathscr{B} \widehat{\otimes} F)^{n+1} \rightarrow 0 \\
& 0 \rightarrow\left(\mathscr{E} \widehat{\otimes} \mathscr{L}^{\prime}\right)^{n} \rightarrow(\mathscr{E} \widehat{\otimes} F)^{n} \rightarrow\left(\mathscr{E} \widehat{\otimes} \mathscr{B}^{\prime}\right)^{n+1} \rightarrow 0 \\
& 0 \rightarrow\left(\mathscr{E} \widehat{\otimes} \mathscr{B}^{\prime}\right)^{n} \rightarrow\left(\mathscr{E} \widehat{\otimes} \mathscr{L}^{\prime}\right)^{n} \rightarrow\left(\mathscr{E} \widehat{\otimes} H^{\prime}\right)^{n} \rightarrow 0 \\
& 0 \rightarrow\left(\mathscr{B} \widehat{\otimes} \mathscr{L}^{\prime}\right)^{n} \rightarrow(\mathscr{B} \widehat{\otimes} F)^{n} \rightarrow\left(\mathscr{B} \widehat{\otimes} \mathscr{B}^{\prime}\right)^{n+1} \rightarrow 0 \\
& 0 \rightarrow\left(\mathscr{B} \widehat{\otimes} \mathscr{B}^{\prime}\right)^{n} \rightarrow\left(\mathscr{B} \widehat{\otimes} \mathscr{E}^{\prime}\right)^{n} \rightarrow\left(\mathscr{B} \widehat{\otimes} H^{\prime}\right)^{n} \rightarrow 0 \\
& 0 \rightarrow\left(\mathscr{\otimes} \widehat{\otimes} H^{\prime}\right)^{n} \rightarrow\left(\mathscr{E} \widehat{\otimes} H^{\prime}\right)^{n} \rightarrow\left(H \widehat{\otimes} H^{\prime}\right)^{n} \rightarrow 0
\end{aligned}
$$

The first one is also an exact sequence of differential complexes when $(\mathscr{E} \widehat{\otimes} F)^{*}$ (respectively $\left.(\mathscr{乃} \widehat{\otimes} F)^{*}\right)$ is endowed with $d^{\prime}=\varepsilon \widehat{\otimes} d_{F}\left(\right.$ respectively $d^{\prime \prime}=-\varepsilon \widehat{\otimes} d_{F}$ ), yielding, by Lemma 2.5 , the long exact sequence

(7) $\cdots \rightarrow H^{*}(\mathscr{E} \widehat{\otimes} F) \rightarrow H^{*}(E \widehat{\otimes} F) \rightarrow H^{*+1}(\mathscr{\otimes} \widehat{\otimes} F) \rightarrow H^{*+1}(\mathscr{E} \widehat{\otimes} F) \rightarrow \cdots$.

Moreover, the sequences (2) and (3) imply that $H^{*}(\mathscr{E} \widehat{\otimes} F) \cong\left(\mathscr{E} \widehat{\otimes} H^{\prime}\right)^{*}$. Indeed the sequence (2) identifies $\left(\mathscr{L} \widehat{\otimes} \mathscr{L}^{\prime}\right)^{*}$ (respectively $\left.\left(\mathscr{L} \widehat{\otimes} \mathscr{B}^{\prime}\right)^{*}\right)$ as being the kernel (respectively the image) of the differential $d^{\prime}$ (the $\varepsilon$ does not affect that conclusion since all maps are graded). Moreover, sequence (3) says that the quotient of $\left(\mathscr{L} \widehat{\otimes} \mathscr{L}^{\prime}\right)^{*}$ by $\left(\mathscr{L} \widehat{\otimes} \mathscr{B}^{\prime}\right)^{*}$ is isomorphic to $\left(\mathscr{L} \widehat{\otimes} H^{\prime}\right)^{*}$. Similarly (4) and (5) imply that $H^{*}(\mathscr{B} \widehat{\otimes} F) \cong\left(\mathscr{B} \widehat{\otimes} H^{\prime}\right)^{*}$. With these isomorphisms, the sequence (7) becomes

$$
\cdots \rightarrow\left(\mathscr{E} \widehat{\otimes} H^{\prime}\right)^{*} \rightarrow H^{*}(E \widehat{\otimes} F) \rightarrow\left(\mathscr{B} \widehat{\otimes} H^{\prime}\right)^{*+1} \stackrel{\nu}{\rightarrow}\left(\mathscr{L} \widehat{\otimes} H^{\prime}\right)^{*+1} \rightarrow \cdots
$$

We will prove that $v$ is the map induced by the natural inclusion $\mathscr{B}^{*} \rightarrow \mathscr{L}^{*}$. Indeed, consider the following diagram:

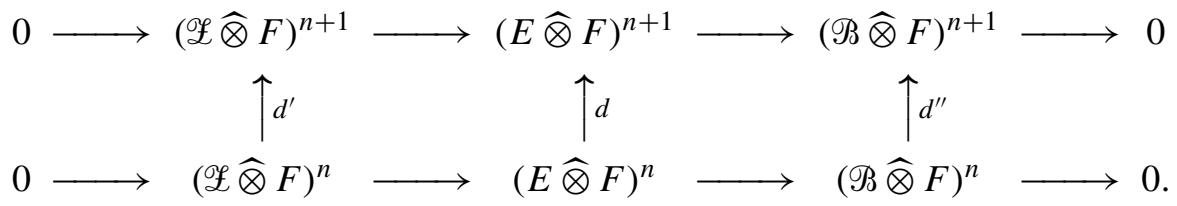

Pick $\sum_{i=1}^{k} b_{i} \otimes z_{i}$ in $\mathscr{B}^{p} \otimes \mathscr{E}^{\prime q}$. Letting $b_{i}=d_{E} x_{i}$ for some $x_{i}$ in $E^{p-1}$, then we have $d\left(\sum_{i=1}^{k} x_{i} \otimes z_{i}\right)=\sum_{i=1}^{k} b_{i} \otimes z_{i}$. This shows that $v$ and $i \widehat{\otimes}$ id coincide on the subspace $\mathscr{B}^{p} \otimes H^{\prime q}$. Therefore they coincide on all of $\mathscr{B} \widehat{\otimes} H^{\prime}$.

Since $v$ is an injective map (again by [T, Proposition 43.7, p. 440]), the long exact sequence (8) is equivalent to the short exact sequence

$$
0 \rightarrow\left(\mathscr{B} \widehat{\otimes} H^{\prime}\right)^{*} \rightarrow\left(\mathscr{E} \widehat{\otimes} H^{\prime}\right)^{*} \rightarrow H^{*}(E \widehat{\otimes} F) \rightarrow 0 .
$$


Hence $H^{*}(E \widehat{\otimes} F) \cong\left(\mathscr{E} \widehat{\otimes} H^{\prime}\right)^{*} /\left(\mathscr{B} \widehat{\otimes} H^{\prime}\right)^{*}$ and the latter space is isomorphic to $H \widehat{\otimes} H^{\prime}$, as the sequence (6) shows.

Proof of Proposition 2.3. The proof is notationally heavy but conceptually quite simple. First observe that the continuous map $\varphi: \Omega^{*}(\mathscr{F}) \otimes \Omega^{*}(\mathscr{G}) \rightarrow \Omega^{*}(\mathscr{F} \times \mathscr{G})$ is injective. We will prove hereafter that it is a homomorphism with dense image, implying that its extension $\varphi: \Omega^{*}(\mathscr{F}) \widehat{\otimes} \Omega^{*}(\varphi) \rightarrow \Omega^{*}(\mathscr{F} \times \mathscr{G})$ is an isomorphism.

To prove that $\varphi$ is a homomorphism, recall that the following subsets of $\Omega^{p}(\mathscr{F})$ form a basis of neighborhoods of 0 :

$$
\begin{aligned}
\mathcal{U}\left(r, \varepsilon,\left\{\left(U_{i}, \phi_{i}\right)\right\},\left\{K_{i}\right\}\right)=\left\{\omega \in \Omega^{p}(\mathscr{F}):\left|D^{a} \omega_{i, j_{1} \cdots j_{p}}(x)\right| \leq \varepsilon\right. \\
\forall \text { multi-index } a=\left(a_{1}, \ldots, a_{\operatorname{dim} M}\right) \text { with }|a| \leq r, \\
\left.\forall 1 \leq i \leq n, \forall x \in K_{i}\right\},
\end{aligned}
$$

where $r$ is some nonnegative integer, $\varepsilon>0,\left\{\left(U_{i}, \phi_{i}\right): 1 \leq i \leq n\right\}$ is a finite collection of foliated charts and $K_{i}$ is a compact subset of $U_{i}$ for each $1 \leq i \leq n$. The functions $\omega_{i, j_{1} \cdots j_{p}}, 1 \leq j_{1}<\cdots<j_{p} \leq \operatorname{dim} M$, denote the tangential coordinates of $\omega$ with respect to the chart $\left(U_{i}, \phi_{i}\right)$ and $D^{a} \omega_{i, j_{1} \cdots j_{p}}$ is the $a$-th derivative

$$
D^{a} \omega_{i, j_{1} \cdots j_{p}}=\frac{\partial^{a_{1}}}{\partial x_{1}} \cdots \frac{\partial^{a_{\operatorname{dim} M}}}{\partial x_{\operatorname{dim} M}}\left(\omega_{i, j_{1} \cdots j_{p}}\right) .
$$

We want to verify that if $U$ is a neighborhood of 0 in $\Omega^{p}(\mathscr{F}) \otimes \Omega^{q}(\mathscr{G})$, then $\varphi(U) \supset$ $O \cap \varphi\left(\Omega^{p}(\mathscr{F}) \otimes \Omega^{q}(\mathscr{G})\right)$ for some neighborhood of 0 in $\Omega^{p+q}(\mathscr{F} \times \mathscr{G})$. Now a neighborhood of 0 in $\Omega^{p}(\mathscr{F}) \otimes \Omega^{q}(\mathscr{G})$ can be chosen of the type

$$
\mathcal{U}\left(U^{o}, V^{o}\right)=\left\{\sum_{i=1}^{I} \alpha_{i} \otimes \beta_{i}: \sup _{\substack{x^{\prime} \in U^{o} \\ y^{\prime} \in V^{o}}}\left|\sum_{i=1}^{I}\left\langle x^{\prime}, \alpha_{i}\right\rangle\left\langle y^{\prime}, \beta_{i}\right\rangle\right| \leq 1\right\},
$$

where $U$ and $V$ are neighborhoods of 0 in $\Omega^{p}(\mathscr{F})$ and $\Omega^{q}(\mathscr{G})$, respectively, and where $U^{o}$ denotes the polar of $U$, that is, the subset

$$
U^{o}=\left\{x^{\prime} \in \Omega^{p}(\mathscr{F})^{\prime}:\left|\left\langle x^{\prime}, u\right\rangle\right| \leq 1 \forall u \in U\right\}
$$

of the dual $\Omega^{p}(\mathscr{F})^{\prime}$ of $\Omega^{p}(\mathscr{F})$ (same for $V^{o}$ ). We say a few words about this issue in the next paragraph.

The (algebraic) tensor product $E \otimes F$ of two locally convex Hausdorff TVS's is isomorphic to $B\left(E_{\sigma}^{\prime}, F_{\sigma}^{\prime}\right)$, the vector space of continuous bilinear forms on the product $E_{\sigma}^{\prime} \times F_{\sigma}^{\prime}$ of the duals of $E$ and $F$ each endowed with its respective weak topology (topology of pointwise convergence) (Proposition 42.4 in [T, p. 432]). The latter space can be naturally realized as a subspace of a complete locally convex Hausdorff TVS, namely the space $\mathscr{B}_{\varepsilon}\left(E_{\sigma}^{\prime}, F_{\sigma}^{\prime}\right)$ of separately continuous bilinear forms on $E_{\sigma}^{\prime} \times F_{\sigma}^{\prime}$ with the $\varepsilon$-topology, or topology of uniform convergence on products of equicontinuous subsets of $E^{\prime}$ and $F^{\prime}$ (Definition 43.1 and 
Proposition 42.3 in [T, pp. 430, 434]). When endowed with the topology induced by $\mathscr{P}_{\varepsilon}\left(E_{\sigma}^{\prime}, F_{\sigma}^{\prime}\right)$, the tensor product of $E$ and $F$ is denoted by

$$
E \otimes_{\varepsilon} F
$$

The topology on $\mathscr{B}_{\varepsilon}\left(E_{\sigma}^{\prime}, F_{\sigma}^{\prime}\right)$ can be defined by the following basis of neighborhoods of 0 :

$$
\mathcal{U}(A, B)=\left\{\phi \in \mathscr{B}_{\varepsilon}\left(E_{\sigma}^{\prime}, F_{\sigma}^{\prime}\right):|\phi(A, B)| \leq 1\right\},
$$

where $A$ (respectively $B$ ) is an equicontinuous subset of $E^{\prime}$ (respectively $F^{\prime}$ ). The reason for the restriction to equicontinuous sets (rather than just bounded sets) is explained in [T, pp. 427-428]. Now any equicontinuous subset of $E^{\prime}$ is contained in the polar $U^{o}$ of some neighborhood $U$ of 0 (Proposition 32.7 in [T, p. 341]). Thus, a basis of neighborhoods of 0 is also given by the sets $U_{\delta}\left(U^{o}, V^{o}\right)$, where $U$ (respectively $V$ ) runs through a basis of neighborhoods of 0 in $E$ (respectively $F$ ).

Returning to the proof that $\varphi$ is a homomorphism, we make the claim that if $U=$ $\mathcal{U}\left(r, \varepsilon,\left\{\left(U_{i}, \phi_{i}\right)\right\},\left\{K_{i}\right\}\right)$ and $V=U\left(s, \delta,\left\{\left(V_{k}, \psi_{k}\right)\right\},\left\{L_{k}\right\}\right)$, then $\varphi\left(U\left(U^{o}, V^{o}\right)\right) \supset$ $\operatorname{Im} \varphi \cap O$, where $O=U\left(\max \{r, s\}, \varepsilon \delta,\left\{\left(U_{i} \times V_{k}, \phi_{i} \times \psi_{k}\right)\right\},\left\{K_{i} \times L_{k}\right\}\right)$. For that purpose, it will be useful to observe that the set $U\left(r, \varepsilon,\left\{\left(U_{i}, \phi_{i}\right)\right\},\left\{K_{i}\right\}\right)$ is the polar of the following subset of the dual of $\Omega^{p}(\mathscr{F})$ :

$$
\begin{aligned}
& \mathscr{A}\left(r, \varepsilon,\left\{\left(U_{i}, \phi_{i}\right)\right\},\left\{K_{i}\right\}\right) \\
& =\left\{\begin{array}{l}
\left\{\ell_{a, \varepsilon, i, j_{1} \cdots j_{p}, x}(\omega)=(1 / \varepsilon) \partial^{a} \omega_{i, j_{1} \cdots j_{p}}(x):\right. \\
\left.\quad|a| \leq r, i=1, \ldots, n, 1 \leq j_{1}<\cdots<j_{p} \leq \operatorname{dim} M, x \in K_{i}\right\} .
\end{array}\right.
\end{aligned}
$$

Thus $U=A^{o}$. On the other hand, a locally convex Hausdorff TVS $E$ is isomorphic to the dual of its weak dual, that is, $E \cong\left(E_{\sigma}^{\prime}\right)^{\prime}$ (Proposition 35.1 in [T, p. 361]), and if $U=A^{o}$, then $U^{o}=\left(A^{o}\right)^{o}$ coincides with the closed convex balanced hull of $A$ (that is, the closure of the convex hull of $\bigcup_{\{\lambda:|\lambda| \leq 1\}} \lambda A$ ), denoted by $\Gamma A$ (Proposition 35.3 in [T, p. 362]). Furthermore, one verifies directly from the definitions involved that $U(A, B)=U(\Gamma A, \Gamma B)$. Thus, $U\left(U^{o}, V^{o}\right)=U(A, B)$ with $A=\mathscr{A}\left(r, \varepsilon,\left\{\left(U_{i}, \phi_{i}\right)\right\},\left\{K_{i}\right\}\right)$ and $B=\mathscr{A}\left(s, \delta,\left\{\left(V_{k}, \psi_{k}\right)\right\},\left\{L_{k}\right\}\right)$.

Now let $\theta=\sum_{t=1}^{T} \alpha_{t} \otimes \beta_{t} \in \Omega^{p}(\mathscr{F}) \otimes \Omega^{q}(\mathscr{G})$ be such that $\varphi(\theta)$ belongs to $O$, that is, for all multi-indices $c$ with $|c| \leq r s$, for all $i, k, j_{1}<\cdots<j_{p}, l_{1}<\cdots<l_{q}$ and for all $(x, y) \in K_{i} \times L_{k}$, one of the following holds:

$$
\begin{gathered}
\left|D^{c}\left(\sum_{t=1}^{T}\left(\alpha_{t}\right)_{i, j_{1} \cdots j_{p}}\left(\beta_{t}\right)_{k, l_{1} \cdots l_{q}}\right)(x, y)\right| \leq \varepsilon \delta, \\
\left|\sum_{t=1}^{T} \frac{1}{\varepsilon} D^{a}\left(\alpha_{t}\right)_{i, j_{1} \cdots j_{p}}(x) \frac{1}{\delta} D^{b}\left(\beta_{t}\right)_{k, l_{1} \cdots l_{q}}(y)\right| \leq 1,
\end{gathered}
$$


where $c=\left(a_{1}, \ldots, a_{\operatorname{dim} M}, b_{1}, \ldots, b_{\operatorname{dim} N}\right)$. Equivalently,

$$
\left|\sum_{t=1}^{T}\left\langle\ell_{a, \varepsilon, i, j_{1} \cdots j_{p}, x}, \alpha_{t}\right\rangle\left\langle\ell_{b, \delta, k, l_{1} \cdots l_{q}, y}, \beta_{t}\right\rangle\right| \leq 1,
$$

which means that $\theta \in \mathcal{U}(A, B)$, thus proving that $\varphi$ is a homomorphism.

It remains to prove that the image of $\varphi$ is dense in $\Omega^{n}\left(\mathscr{F}_{\times} \mathscr{G}\right)$. This is essentially a consequence of the fact that polynomial functions are dense in the space of smooth functions on the Euclidean space, implying that if $X$ and $Y$ are open subsets of $\mathbb{R}^{n}$ and $\mathbb{R}^{m}$ respectively, then the tensor product $C_{c}^{\infty}(X) \otimes C_{c}^{\infty}(Y)$ of the spaces of smooth functions with compact supports on $X$ and $Y$ is dense in the space $C^{\infty}(X \times Y)$ of smooth functions on $X \times Y$ (see [T, Theorem 39.2, p. 409 and Corollary 1, p. 159]). Let $\omega \in \Omega^{n}(\mathscr{F} \times \mathscr{G})$ and consider $U$ a neighborhood of $\omega$ of the type $\omega+\mathcal{U}\left(r, \varepsilon,\left\{\left(U_{i} \times V_{k}, \phi_{i} \times \psi_{k}\right),\left\{K_{i} \times L_{k}\right\}\right\}\right)$. For each tangential component $\omega_{i, k, j_{1} \cdots j_{p}, l_{1} \cdots l_{q}}$, denoted hereafter $\omega_{i, k, J, L}$, of $\omega$ with respect to the chart $\phi_{i} \times \psi_{k}$, pick functions $f_{i, k, J, L}^{n} \in C_{c}^{\infty}\left(U_{i}\right)$ and $g_{i, k, J, L}^{n} \in C_{c}^{\infty}\left(V_{k}\right), n=1, \ldots, N$, such that

$$
\sum_{n=1}^{N} f_{i, k, J, L}^{n} g_{i, k, J, L}^{n}
$$

lies in $\omega_{i, k, J, L}+\mathcal{U}\left(r, \varepsilon, K_{i} \times L_{k}\right) \subset C^{\infty}\left(U_{i} \times V_{k}\right)$. Then the form

$$
\sum_{n, J, L} f_{i, k, J, L}^{n} g_{i, k, J, L}^{n} d x^{J} \wedge d x^{L}
$$

belongs to $U \cap \varphi\left(\Omega^{p}(\mathscr{F}) \otimes \Omega^{q}(\mathscr{G})\right)$.

\section{Künneth formula when one of the factors is finite-dimensional}

Another natural question is whether the Künneth formula holds in the case where the tensor product does not need to be completed, that is, when one of the factors, say $H^{*}(\mathscr{F})$, is finite-dimensional. The answer is positive provided the ambient manifold of that factor is compact. There is no assumption on the second factor. This statement was already well-known when $\mathscr{F}$ is a one-leaf foliation (see [El Kacimi-Alaoui 1983; Moore and Schochet 2006]). We use the fact that under the previous assumptions, the foliated de Rham differential $d_{\mathscr{F}}$ admits a right inverse, which is implied by results in the theory of splitting of exact sequences of Fréchet spaces appearing in [Meise and Vogt 1997; Vogt 2004].

Proposition 3.1. Let $(M, \mathscr{F})$ and $(N, \mathscr{G})$ be foliated manifolds. Suppose $H^{*}(\mathscr{F})$ is finite-dimensional and $M$ is compact. Then, as topological vector spaces,

$$
H^{*}(\mathscr{F} \times \mathscr{G}) \cong H^{*}(\mathscr{F}) \otimes H^{*}(\mathscr{G}) .
$$


Although we may not anymore quote theorems about coincidence of the $\varepsilon$ - and $\pi$-topologies on $H^{*}(\mathscr{F}) \otimes H^{*}(\mathscr{G})$ since $H^{*}(\mathscr{G})$ might not be Hausdorff, one may verify directly that both these topologies coincide with the direct sum topology that appears when $H^{*}(\mathscr{F}) \otimes H^{*}(\mathscr{G})$ is identified with a finite direct sum $\bigoplus_{i=1}^{n} H^{*}(\mathscr{G})$ via the choice of a basis of $H^{*}(\mathscr{F})$.

Remark 3.2. The assumption that $H^{*}(\mathscr{F})$ is finite-dimensional implies that it is Hausdorff as well. This follows from the well-known fact that for a continuous linear map $A: E \rightarrow F$ between Fréchet spaces, if the range is finite-codimensional it is also closed, itself a consequence of the open mapping theorem. Indeed, let $f_{1}, \ldots, f_{n}$ be a basis of an algebraic complement to $\operatorname{Im} A$ in $F$. Define the map

$$
A^{\prime}: E / \operatorname{Ker} A \oplus \mathbb{R}^{n} \rightarrow F, \quad\left([e], a_{1}, \ldots, a_{n}\right) \mapsto A(e)+a_{1} f_{1}+\cdots+a_{n} f_{n} .
$$

It is continuous as the sum of two continuous maps and bijective. Hence it is an isomorphism. Since $\operatorname{Im} A$ is the image of the closed subspace $E / \operatorname{Ker} A$, it is closed as well. We thank the referee for pointing this out to us.

Proof of Proposition 3.1. The idea is to replace the complex $\left(\Omega^{*}(\mathscr{F}), d_{\mathscr{F}}\right)$ by a homotopy equivalent finite-dimensional complex $\left(V, d_{V}\right)$. It is then easy to prove that $H^{*}\left(V \otimes \Omega^{*}(\varphi)\right)$ coincides with $H^{*}\left(\Omega^{*}(\mathscr{F}) \widehat{\otimes} \Omega^{*}(\varphi)\right)=H^{*}(\mathscr{F} \times \mathscr{G})$. Besides, it is well-known that $H^{*}\left(V \otimes \Omega^{*}(\mathscr{G})\right)=H^{*}(V) \otimes H^{*}\left(\Omega^{*}(\mathscr{G})\right)$ as vector spaces and it is not difficult to be convinced that this equality holds for the topologies as well. So we are done. To obtain an equivalence with a finite-dimensional complex we need a right inverse for the foliated differential $d_{\mathscr{F}}$, that is, a continuous linear map $\varphi: \mathscr{B}^{*+1}(\mathscr{F}) \rightarrow \Omega^{*}(\mathscr{F})$ such that $d_{\mathscr{F}} \circ \varphi=$ id. This is the content of the Lemma 3.3 below. We assume this fact and proceed with the present proof.

The complex $\left(\Omega^{*}(\mathscr{F}), d_{\mathscr{F}}\right)$ is denoted hereafter by $(\Omega, d)$, Ker $d_{\mathscr{F}}$ by $\mathscr{L}$ and $\operatorname{Im} d \mathscr{F}$ by $\mathscr{B}$. Consider closed foliated forms (of pure degree) $\alpha_{1}, \ldots, \alpha_{n}$ representing a basis $\left\{\left[\alpha_{1}\right], \ldots,\left[\alpha_{n}\right]\right\}$ of $H^{*}(\mathscr{F})$. The subset $V=\left\{\alpha_{1}, \ldots, \alpha_{n}\right\}$ endowed with the zero differential $\left(d_{V}=0\right)$ is a finite-dimensional subcomplex of $(\Omega, d)$ with cohomology $H^{*}(\mathscr{F})$. It is thus (algebraically) homotopy equivalent to $(\Omega, d)$ (see [Spanier 1966, Theorem 7.4.10, p. 192]). We show next that the homotopy, its inverse and the equivalence may be chosen continuous when $d$ admits a right inverse.

We first need to set up some notation.

- The natural inclusion $V \rightarrow \Omega$ is denoted by $i$.

- The map $\varphi: \mathscr{B} \rightarrow \Omega$ denotes a continuous linear right inverse to $d$.

- The cohomology class of a closed form $\beta$ is denoted by $[\beta]$.

- Since $H^{*}(\mathscr{F})$ is finite-dimensional and Hausdorff (see Remark 3.2), the linear map $e: H^{*}(\mathscr{F}) \rightarrow V$ such that $e\left(\left[\alpha_{i}\right]\right)=\alpha_{i}$ is continuous; it is a right inverse for the natural projection $\mathscr{L}^{*}(\mathscr{F}) \rightarrow H^{*}(\mathscr{F})$ with values in $V$. 
Define $\sigma: \Omega \rightarrow V$ and $D: \Omega \rightarrow \Omega$ by

$$
\begin{aligned}
& \sigma(\beta)=e[\beta-\varphi(d \beta)], \\
& D(\beta)=-\varphi((\beta-\varphi(d \beta))-i \circ e[\beta-\varphi(d \beta)]) .
\end{aligned}
$$

The maps $\sigma$ and $D$ are clearly continuous. It is only necessary to verify that $\sigma$ is a cochain map, that $\sigma \circ i=\mathrm{id}_{V}$ and that $i \circ \sigma=\mathrm{id}_{\Omega}+D \circ d+d \circ D$. The first two assertions are obvious and the third holds since

$$
\begin{aligned}
(D \circ d+d \circ D)(\beta) & =-\varphi(d \beta)-d \circ \varphi((\beta-\varphi(d \beta))-i \circ e[\beta-\varphi(d \beta)]) \\
& =-\beta+i \circ e[\beta-\varphi(d \beta)]=-\beta+i \circ \sigma(\beta) .
\end{aligned}
$$

Now the continuous cochain maps $i$ and $\sigma$ induce continuous cochain maps $i \widehat{\otimes}$ id: $V \widehat{\otimes} \Omega^{*}(\mathscr{G}) \rightarrow \Omega^{*}(\mathscr{F}) \widehat{\otimes} \Omega^{*}(\mathscr{G})$ and $\sigma \widehat{\otimes} \mathrm{id}: \Omega^{*}(\mathscr{F}) \widehat{\otimes} \Omega^{*}(\mathscr{G}) \rightarrow V \widehat{\otimes} \Omega^{*}(\mathscr{G})$ such that

$$
\begin{aligned}
& (\sigma \widehat{\otimes} \mathrm{id}) \circ(i \widehat{\otimes} \mathrm{id})=\mathrm{id}, \\
& (i \widehat{\otimes} \mathrm{id}) \circ(\sigma \widehat{\otimes} \mathrm{id})=\mathrm{id}+D^{\prime} \circ d_{\mathscr{F} \times \mathscr{G}}+d_{\mathscr{F} \times \mathscr{G}} \circ D^{\prime},
\end{aligned}
$$

where $D^{\prime}=D \widehat{\otimes} \mathrm{id}$ and $d_{\mathscr{F} \times} \times{ }_{\varphi}=d_{\mathscr{F}} \widehat{\otimes} \mathrm{id}+\varepsilon \widehat{\otimes} d \varphi$. (The last assertion follows from the fact that $\varepsilon \circ D+D \circ \varepsilon=0$.) Furthermore, the continuous cochain maps $i \widehat{\otimes}$ id and $\sigma \widehat{\otimes}$ id induce continuous maps on cohomology that are inverse to one another. This shows that $H^{*}\left(V \widehat{\otimes} \Omega^{*}(\mathscr{G})\right) \cong H^{*}(\mathscr{F} \times \mathscr{G})$ as TVS's. Of course, $V \widehat{\otimes} \Omega^{*}(\mathscr{G})$ is the same as $V \otimes \Omega^{*}(\mathscr{G})$ since $V \otimes E$ is complete when $V$ is finite-dimensional and $E$ is complete.

Finally, the fact that $H^{*}\left(V \otimes \Omega^{*}(\mathscr{G})\right) \cong V \otimes H^{*}(\mathscr{G})$ follows from considering the short exact sequences

$$
\begin{aligned}
& 0 \longrightarrow V \otimes \mathscr{L}^{*}(\mathscr{G}) \longrightarrow V \otimes \Omega^{*}(\mathscr{G}) \stackrel{\varepsilon \otimes d_{\mathscr{G}}}{\longrightarrow} V \otimes \mathscr{B}^{*+1}(\mathscr{G}) \longrightarrow 0, \\
& 0 \longrightarrow V \otimes \mathscr{B}^{*}(\mathscr{G}) \longrightarrow V \otimes \mathscr{L}^{*}(\mathscr{G}) \longrightarrow V \otimes H^{*}(\mathscr{G}) \longrightarrow 0 .
\end{aligned}
$$

Observe that $\varepsilon \otimes d \varphi$ is continuous but not open. Likewise $V \otimes \mathscr{B}^{*+1}(\mathscr{G})$ is not complete and $V \otimes H^{*}(\mathscr{G})$ is not Hausdorff. Nevertheless, the first sequence tells us that the kernel of the differential $\varepsilon \otimes d \varphi$ on $V \otimes \Omega^{*}(\varphi)$ is $V \otimes \mathscr{L}^{*}(\varphi)$ and that its image is $V \otimes \mathscr{B}^{*+1}(\mathscr{G})$. Besides, the topology induced on $V \otimes \mathscr{L}^{*}(\mathscr{G})$ (respectively $V \otimes \mathscr{B}^{*+1}(\mathscr{G})$ ) from its embedding in $V \otimes \Omega^{*}(\mathscr{G})$ coincides with the tensor product topology (Proposition 43.7 in [T, p. 440]). Finally, since the maps in the second sequence are homomorphisms (remembering that $V \otimes H^{*}(\varphi)$ carries the direct sum topology), the quotient $V \otimes \mathscr{L}^{*}(\mathscr{G}) / V \otimes \mathscr{B}^{*}(\mathscr{G})$ is isomorphic to $V \otimes H^{*}(\mathscr{G})$.

Lemma 3.3. If $M$ is compact and $H^{*}(\mathscr{F})$ is finite-dimensional then $d_{\mathscr{F}}$ admits a continuous linear right inverse.

Proof. The proof relies on the following result. 
Theorem 3.4 [Meise and Vogt 1997, Splitting theorem 30.1, p. 378]. Let E, F, G be Fréchet-Hilbert spaces and let $0 \rightarrow F \rightarrow G \rightarrow E \rightarrow 0$ be a short exact sequence of continuous linear maps. If $E$ has property $(\mathrm{DN})$ and $F$ has property $(\Omega)$, then the sequence splits.

We explain hereafter why Theorem 3.4 can be applied to the short exact sequence

$$
0 \rightarrow \mathscr{L}^{*}(\mathscr{F}) \rightarrow \Omega^{*}(\mathscr{F}) \rightarrow \mathscr{B}^{*}(\mathscr{F}) \rightarrow 0 .
$$

The assumption that the spaces are Fréchet-Hilbert is automatically satisfied for nuclear Fréchet spaces (see [Meise and Vogt 1997, Definition, p. 370 and Lemma 28.1 , p. 344]). We mention the definitions of properties (DN) and $(\Omega)$ for completeness but we will only need here the fact that they are stable under performing certain operations. Let $E$ be a Fréchet space endowed with a countable fundamental systems $\left\{\|\cdot\|_{k}: k \geq 1\right\}$ of seminorms (that is, for all $x \in E, x \neq 0$, there exists $k$ such that $\|x\|_{k}>0$ and for all $k_{1}, k_{2}$, there exists $k_{3}$ and a constant $C$ such that $\max \left\{\|\cdot\|_{k_{1}},\|\cdot\|_{k_{2}}\right\} \leq C\|\cdot\|_{k_{3}}$ ). The property (DN) is satisfied by $E$ if and only if it supports a continuous norm $\|\cdot\|$ on $E$ such that for any seminorm $\|\cdot\|_{k}$ there exists a constant $C$ and a seminorm $\|\cdot\|_{K}$ such that

$$
\|x\|_{k}^{2} \leq C\|x\|\|x\|_{K} \quad \text { for all } x \in E .
$$

The property $(\Omega)$ is satisfied by $E$ if and only if for each $p \geq 1$ there exists a $q \geq 1$ so that for every $k \geq 1$, there exists a $0<\theta<1$ and a constant $C$ such that

$$
\|y\|_{q}^{*} \leq C\|y\|_{p}^{* 1-\theta}\|y\|_{k}^{* \theta} \quad \text { for all } y \in E^{\prime},
$$

where $\|y\|_{k}^{*}$ means $\sup \left\{|y(x)|:\|x\|_{k} \leq 1\right\}$.

Both properties are satisfied by the Schwartz space

$$
s=\left\{\left(x_{j}\right)_{j \geq 1}: \sum_{j=1}^{\infty}\left|x_{j}\right|^{2} j^{2 k}<\infty \forall k \geq 1\right\}
$$

[Meise and Vogt 1997, Example 29.5(1), p. 363, Lemma 29.2, p. 359 and Lemma 29.11, p. 368]. Any space of $C^{\infty}$-sections of a finite-dimensional vector bundle $E$ over a compact manifold is isomorphic, as topological vector space, to $s$ (see [Valdivia 1982] ${ }^{1}$.

${ }^{1}$ The reference [Valdivia 1982] contains a proof of the fact that for a compact manifold $M$, the space $C^{\infty}(M) \cong s$ which can easily be adjusted to the case of $C^{\infty}(M, E)$. Indeed, a finite partition of unity $\left\{\theta_{i}: i=1, \ldots, n\right\}$ subordinated to a cover of $M$ by trivializing open subsets allows us to identify the space of smooth sections of the bundle $E$ with a finite direct sum $\bigoplus_{i} C_{c}^{\infty}\left(C_{i}, \mathbb{R}^{d}\right)$, where $C_{i}$ is the support of $\theta_{i}$, where $d$ is the rank of $E$ and where $C_{c}^{\infty}\left(C_{i}, \mathbb{R}^{d}\right)$ is the set of smooth functions with compact support in $C_{i}$. Because each $C_{c}^{\infty}\left(C_{i}, \mathbb{R}\right)$ is isomorphic to $s$ [Valdivia 1982, (5), p. 536] and $s \oplus s \cong s$ [Valdivia 1982, (5), p. 327], we reach our conclusion. 
Thus the space of foliated forms $\Omega^{*}(\mathscr{F})$ enjoys the properties (DN) and $(\Omega)$. Property (DN) is inherited by closed subspaces (see [Meise and Vogt 1997, Lemma 29.2 , p. 359]). So $\mathscr{B}^{*}(\mathscr{F})$ has property $(\mathrm{DN})$. To see that $\mathscr{L}^{*}(\mathscr{F})$ has property $(\Omega)$, we use the fact that $H^{*}(\mathscr{F})$ is finite-dimensional. Indeed, property $(\Omega)$ is inherited by quotients by closed subspaces (see [Meise and Vogt 1997, Lemma 29.11(2), p. 368]) so that $\mathscr{B}^{*}(\mathscr{F})$, which is isomorphic to $\Omega^{*-1}(\mathscr{F}) / \mathscr{E}^{*-1}(\mathscr{F})$, has property $(\Omega)$. Since $H^{*}(\mathscr{F})$ is finite-dimensional, the natural projection $\mathscr{L}^{*}(\mathscr{F}) \rightarrow H^{*}(\mathscr{F})$ admits a right inverse so that $\mathscr{L}^{*}(\mathscr{F}) \cong \mathscr{B}^{*}(\mathscr{F}) \oplus H^{*}(\mathscr{F})$ and can be thought of as a quotient of $\Omega^{*-1}(\mathscr{F}) \oplus H^{*}(\mathscr{F})$ (by $\mathscr{L}^{*-1}(\mathscr{F}) \oplus\{0\}$ ), which is itself also isomorphic to the Schwartz space $s$ when $* \geq 1$. Finally, $\mathscr{L}^{0}(\mathscr{F})$ has property $(\Omega)$ because it is finite-dimensional (it is thus a Banach space).

Remark 3.5. When the manifold $M$ is not compact, the space $\Omega^{*}(\mathscr{F})$ is isomorphic to $s^{\mathbb{N}}$ (argument similar to the compact case with a locally finite partition of unity subordinated to an open cover of $M$ by foliated chart domains) rather than $s$. So that it does not anymore enjoy properties (DN) nor $(\Omega)$, but rather, so-called properties $\left(\mathrm{DN}_{\mathrm{loc}}\right)$ and $\left(\Omega_{\mathrm{loc}}\right)$. One might nevertheless reach a similar conclusion, using the following splitting theorem due to Vogt, if one could prove that $d_{\mathscr{F}}$ is a SK-homomorphism when it is a homomorphism (this does not hold in general, but might be true for $d_{\mathscr{F}}$ ).

Theorem 3.6 [Vogt 2004, Theorem 3.5, p. 820]. Let $0 \rightarrow F \rightarrow G \rightarrow E \rightarrow 0$ be an exact sequence of nuclear Fréchet spaces, $A$ an $S K$-homomorphism. If $E$ has property $\left(\mathrm{DN}_{\mathrm{loc}}\right)$ and $F$ property $\left(\Omega_{\mathrm{loc}}\right)$, then the sequence splits.

\section{Counterexample}

One would like to understand what happens when neither of the situations encountered above occurs. Let $(M, \mathscr{F})$ and $(N, \mathscr{G})$ be two foliated manifolds. Suppose that both foliated cohomologies are infinite-dimensional with one of them nonHausdorff. Then the tensor product $H^{*}(\mathscr{F}) \otimes H^{*}(\mathscr{G})$ cannot be completed. There is nevertheless a case where an alternative to completion could be proposed, that is, when one of the foliations, say $\mathscr{G}$, is a foliation by points $\mathscr{G}_{=} \mathscr{F}_{N}$. Then $H^{*}(\mathscr{G})$ coincides with $C^{\infty}(N)$ and one is tempted to replace $H^{*}(\mathscr{F}) \widehat{\otimes} C^{\infty}(N)$, which does not make sense here, by $C^{\infty}\left(N, H^{*}(\mathscr{F})\right)$, since these two spaces coincide when $H^{*}(\mathscr{F})$ is Hausdorff (Theorem 44.1 in [T, p. 449]). It is therefore natural to wonder whether the trivial map $H^{*}\left(\mathscr{F} \times \mathscr{F}_{N}\right) \rightarrow C^{\infty}\left(N, H^{*}\left(\mathscr{F}_{N}\right)\right)$ yields an isomorphism or not:

$$
H^{*}\left(\mathscr{F} \times \mathscr{F}_{N}\right) \stackrel{?}{\cong} C^{\infty}\left(N, H^{*}(\mathscr{F})\right) .
$$

The answer is negative. Indeed, the torus $\mathbb{T}^{2}$ endowed with a Liouville foliation (see Example 1.2) supports a smooth family of foliated exact forms which is not 
the coboundary of any smooth, nor even continuous, family of forms. This smooth family represents thus both the zero element in $C^{\infty}\left(N, H^{*}(\mathscr{F})\right)$ and a nonzero element in $H^{*}\left(\mathscr{F} \times \mathscr{F}_{N}\right)$. I do not see any obvious theoretical reason for such a family to exist; both the space $H^{*}\left(\mathscr{F}_{F} \times \mathscr{F}_{N}\right)$ and the space $C^{\infty}\left(N, H^{*}(\mathscr{F})\right)$ are non-Hausdorff; somehow $H^{*}\left(\mathscr{F} \times \mathscr{F}_{N}\right)$ is "more separated" than $C^{\infty}\left(N, H^{*}(\mathscr{F})\right)$.

Let $x, y$ denote standard coordinates on the torus $\mathbb{T}^{2}$. The leaves of the foliation $\mathscr{F}_{\alpha}$ are the orbits of the vector field $X=\partial_{x}+\alpha \partial_{y}$. Any foliated 1-form is automatically closed and can be written $f \overline{d x}$, with $f$ in $C^{\infty}\left(\mathbb{T}^{2}\right)$ and $\overline{d x}$ the image of the closed form $d x \in \Omega^{1}\left(\mathbb{T}^{2}\right)$ in $\Omega^{1}\left(\mathscr{F}_{\alpha}\right)$. It is exact when $f \overline{d x}=\overline{d g}$ for some $g$ in $C^{\infty}\left(\mathbb{T}^{2}\right)$, which is equivalent to $f=X g$. Besides, we may consider the Fourier expansions of the functions $f$ and $g$ :

$$
f=\sum_{m, n \in \mathbb{Z}} f_{m, n} e^{2 \pi i(m x+n y)} \quad \text { and } \quad g=\sum_{m, n \in \mathbb{Z}} g_{m, n} e^{2 \pi i(m x+n y)} .
$$

The equation $f=X g$ is equivalent to the sequence of equations

$$
f_{m, n}=2 \pi i(m+\alpha n) g_{m, n}, \quad m, n \in \mathbb{Z},
$$

which of course implies $f_{0,0}=0$.

Lemma 4.1. Suppose that $\left(f_{t}\right)_{t \in \mathbb{R}}$ is a family of functions on $\mathbb{T}^{2}$. It is a smooth family of smooth functions if and only if each function $t \mapsto\left(f_{t}\right)_{m, n}$ is smooth and for all compact intervals $I$ in $\mathbb{R}$ and integers $a \geq 0, j \geq 1$, there exists a constant $c=c(I, a, j)$ such that

$$
\sup _{t \in I}\left|\partial_{t}^{a}\left(f_{t}\right)_{m, n}\right| \leq \frac{c}{(|m|+|n|)^{j}} .
$$

To see the necessity of this condition it suffices to combine integration by parts in order to get rid of the derivatives with respect to $x$ and $y$ with the fact that the Fourier coefficient $\left(\partial_{x}^{k} \partial_{y}^{l} \partial_{t}^{a} f_{t}\right)_{m, n}$ is bounded by a constant depending only on $I, k, l$ and $a$.

With these preliminaries in mind, we are ready to construct a family $f_{t}$ of functions on $\mathbb{T}^{2}$ with the following properties:

(i) $f_{t}$ is a smooth family of smooth functions.

(ii) For each value of the parameter $t$, there is a smooth solution to $f_{t}=X g_{t}$.

(iii) There no smooth, or even continuous, family of smooth functions $g_{t}$ solving $f_{t}=X g_{t}$.

Since $\alpha$ is a Liouville number, for each integer $p>1$, there exists a pair of integers $\left(m_{p}, n_{p}\right)$ such that

$$
\left|m_{p}+\alpha n_{p}\right| \leq \frac{1}{\left(\left|m_{p}\right|+\left|n_{p}\right|\right)^{p}}
$$


Without loss of generality assume that $\left(m_{p}, n_{p}\right) \neq\left(m_{q}, n_{q}\right)$ for $p \neq q$ and that $n_{p} \geq p$. Now define

$$
\left(f_{t}\right)_{m, n}= \begin{cases}\left(m_{p}+\alpha n_{p}\right)\left(\left|m_{p}\right|+\left|n_{p}\right|\right) \rho\left(s_{p}\left(t-\frac{1}{p}\right)\right) & \text { if }(m, n)=\left(m_{p}, n_{p}\right) \\ 0 & \text { otherwise }\end{cases}
$$

where $\rho$ is a bump function supported in the interval $[-1,1]$ that achieves its maximum value 1 at 0 and where $s_{p}=p(p+1)$. The function $\rho\left(s_{p}\left(t-\frac{1}{p}\right)\right)$ has its support contained in

$$
\left[\frac{1}{p}-\frac{1}{2 p(p+1)}, \frac{1}{p}+\frac{1}{2 p(p+1)}\right] .
$$

We verify that the $\left(f_{t}\right)_{m, n}$ 's are the Fourier coefficients of a family $f_{t}$ enjoying properties (i), (ii) and (iii).

(i) For smoothness of $f_{t}$ we use the criterion described in Lemma 4.1.

$$
\begin{aligned}
\left|\partial_{t}^{a}\left(f_{t}\right)_{m_{p}, n_{p}}\right| & \leq\left|\left(m_{p}+\alpha n_{p}\right)\right|\left(\left|m_{p}\right|+\left|n_{p}\right|\right) \sup _{t \in I}\left|\partial_{t}^{a} \rho(t)\right|\left|s_{p}\right|^{a} \\
& \leq \frac{c_{a}\left|s_{p}\right|^{a}}{\left(\left|m_{p}\right|+\left|n_{p}\right|\right)^{p-1}} \leq \frac{c_{a}^{\prime}}{\left(\left|m_{p}\right|+\left|n_{p}\right|\right)^{p-1-2 a}} \leq \frac{c_{a, j}^{\prime \prime}}{\left(\left|m_{p}\right|+\left|n_{p}\right|\right)^{j}} .
\end{aligned}
$$

The second inequality on the last line follows from the fact that $s_{p}$ is a polynomial of degree 2 in $p$ and the assumption $n_{p} \geq p$, while the last inequality is a consequence of the fact that $p-1-2 a \rightarrow \infty$ when $p \rightarrow \infty$.

(ii) The coefficients $\left(g_{t}\right)_{m, n}=\left(f_{t}\right)_{m, n} /(m+\alpha n)$ define a smooth function for each value of $t$. Indeed, for a fixed $t_{0}$,

$$
\left(g_{t_{0}}\right)_{m_{p}, n_{p}}=\left(\left|m_{p}\right|+\left|n_{p}\right|\right) \rho\left(s_{p}\left(t_{0}-1 / p\right)\right)=0
$$

for all $p$ except perhaps one since the supports of the various functions $\rho\left(s_{p}\left(t_{0}-\frac{1}{p}\right)\right)$ are disjoint. The Fourier series of the function $g_{t_{0}}$ has thus only one term.

(iii) The function $g_{t}$ is not smooth, nor even continuous, near $t=0$. Indeed, the coefficients $\left(g_{t}\right)_{m, n}$ are not uniformly bounded on any interval $I$ around 0 :

$$
\sup _{t \in I}\left|\left(g_{t}\right)_{m_{p}, n_{p}}\right|=\left(\left|m_{p}\right|+\left|n_{p}\right|\right) \sup _{t \in I}\left|\rho\left(s_{p}\left(t_{0}-1 / p\right)\right)\right|=\left(\left|m_{p}\right|+\left|n_{p}\right|\right)
$$

as soon as $p$ is sufficiently large for $\frac{1}{p}$ to belong to $I$.

\section{Acknowledgments}

I wish to thank warmly Professor Dietmar Vogt for helping me to clarify how I could use his results on splittings of exact sequences of Fréchet spaces explained in [Meise and Vogt 1997; Vogt 2004]. I also wish to thank Alan Weinstein and 
Pierre Bieliavsky for useful discussions regarding the subject. Finally, I am very much indebted to the referee who noticed a major gap in the initial manuscript and made very useful comments.

\section{References}

[Bertelson 2001] M. Bertelson, "Foliations associated to regular Poisson structures", Commun. Contemp. Math. 3:3 (2001), 441-456. MR 2002i:53110 Zbl 1002.53056

[Bredon 1997] G. E. Bredon, Sheaf theory, 2nd ed., Graduate Texts in Math. 170, Springer, New York, 1997. MR 98g:55005 Zbl 0874.55001

[El Kacimi-Alaoui 1983] A. El Kacimi-Alaoui, "Sur la cohomologie feuilletée", Compositio Math. 49:2 (1983), 195-215. MR 85a:57016 Zbl 0516.57017

[Grothendieck 1955] A. Grothendieck, Produits tensoriels topologiques et espaces nucléaires, Mem. Amer. Math. Soc. 16, Amer. Math. Soc., Providence, RI, 1955. MR 17,763c Zbl 0123.30301

[Haefliger 1980] A. Haefliger, "Some remarks on foliations with minimal leaves", J. Differential Geom. 15:2 (1980), 269-284 (1981). MR 82j:57027 Zbl 0444.57016

[Meise and Vogt 1997] R. Meise and D. Vogt, Introduction to functional analysis, Oxford Graduate Texts in Math. 2, Oxford Univ. Press, New York, 1997. MR 98g:46001 Zbl 0924.46002

[Moore and Schochet 2006] C. C. Moore and C. L. Schochet, Global analysis on foliated spaces, 2nd ed., MSRI Publ. 9, Cambridge Univ. Press, New York, 2006. MR 2006i:58035 Zbl 1091.58015

[Schwartz 1954] L. Schwartz, "Opérations algébriques sur les distributions à valeur vectorielle: Théorème de Künneth”, pp. 1-6, Exp. No. 24 in Séminaire Schwartz de la Faculté des Sciences de Paris, 1953/1954: Produits tensoriels topologiques d'espaces vectoriels topologiques, Espaces vectoriels topologiques nucléaires, Applications, Sec. math., Paris, 1954. MR 17,764a Zbl 0059.10401

[Spanier 1966] E. H. Spanier, Algebraic topology, McGraw-Hill, New York, 1966. MR 35 \#1007 Zbl 0145.43303

[Trèves 1967] F. Trèves, Topological vector spaces, distributions and kernels, Academic Press, New York, 1967. MR 37 \#726 Zbl 0171.10402

[Valdivia 1982] M. Valdivia, Topics in locally convex spaces, North-Holland Math. Studies 67, Notas de Mat. 85, North-Holland, Amsterdam, 1982. MR 84i:46007 Zbl 0489.46001

[Vogt 2004] D. Vogt, "Splitting of exact sequences of Fréchet spaces in the absence of continuous norms", J. Math. Anal. Appl. 297:2 (2004), 812-832. Special issue dedicated to John Horváth. MR 2005i:35029 Zbl 1065.46003

Received April 10, 2008. Revised March 9, 2011.

MÉlanie BERTELSON

DÉPARTEMENT DE MATHÉMATIQUES

UNIVERSITÉ LIBRE DE BRUXELLES

ULB, CP 218

BOULEVARD DU TRIOMPHE

B-1050 BRUXELLES

BELGIUM

mbertels@ulb.ac.be 


\title{
PACIFIC JOURNAL OF MATHEMATICS
}

\author{
http://www.pjmath.org \\ Founded in 1951 by
}

E. F. Beckenbach (1906-1982) and F. Wolf (1904-1989)

\section{EDITORS}

V. S. Varadarajan (Managing Editor)

Department of Mathematics

University of California

Los Angeles, CA 90095-1555

pacific@math.ucla.edu

Vyjayanthi Chari

Department of Mathematics

University of California

Riverside, CA 92521-0135

chari@math.ucr.edu

\section{Robert Finn}

Department of Mathematics Stanford University

Stanford, CA 94305-2125

finn@math.stanford.edu

Kefeng Liu

Department of Mathematics

University of California

Los Angeles, CA 90095-1555

liu@math.ucla.edu
Darren Long

Department of Mathematics

University of California

Santa Barbara, CA 93106-3080

long@math.ucsb.edu

Jiang-Hua Lu

Department of Mathematics

The University of Hong Kong

Pokfulam Rd., Hong Kong jhlu@maths.hku.hk

Alexander Merkurjev

Department of Mathematics University of California

Los Angeles, CA 90095-1555 merkurev@math.ucla.edu
Sorin Popa

Department of Mathematics

University of California

Los Angeles, CA 90095-1555

popa@math.ucla.edu

Jie Qing

Department of Mathematics

University of California

Santa Cruz, CA 95064

qing@ cats.ucsc.edu

Jonathan Rogawski

Department of Mathematics

University of California

Los Angeles, CA 90095-1555

jonr@math.ucla.edu

\section{PRODUCTION}

pacific@math.berkeley.edu

Silvio Levy, Scientific Editor Matthew Cargo, Senior Production Editor

ACADEMIA SINICA, TAIPEI

CALIFORNIA INST. OF TECHNOLOGY

INST. DE MATEMÁTICA PURA E APLICADA

KEIO UNIVERSITY

MATH. SCIENCES RESEARCH INSTITUTE

NEW MEXICO STATE UNIV.

OREGON STATE UNIV.

\section{SUPPORTING INSTITUTIONS}

STANFORD UNIVERSITY
UNIV. OF BRITISH COLUMBIA
UNIV. OF CALIFORNIA, BERKELEY
UNIV. OF CALIFORNIA, DAVIS
UNIV. OF CALIFORNIA, LOS ANGELES
UNIV. OF CALIFORNIA, RIVERSIDE
UNIV. OF CALIFORNIA, SAN DIEGO
UNIV. OF CALIF., SANTA BARBARA

UNIV. OF CALIF., SANTA CRUZ

UNIV. OF MONTANA

UNIV. OF OREGON

UNIV. OF SOUTHERN CALIFORNIA

UNIV. OF UTAH

UNIV. OF WASHINGTON

WASHINGTON STATE UNIVERSITY

These supporting institutions contribute to the cost of publication of this Journal, but they are not owners or publishers and have no responsibility for its contents or policies.

See inside back cover or www.pjmath.org for submission instructions.

The subscription price for 2011 is US \$420/year for the electronic version, and \$485/year for print and electronic.

Subscriptions, requests for back issues from the last three years and changes of subscribers address should be sent to Pacific Journal of Mathematics, P.O. Box 4163, Berkeley, CA 94704-0163, U.S.A. Prior back issues are obtainable from Periodicals Service Company, 11 Main Street, Germantown, NY 12526-5635. The Pacific Journal of Mathematics is indexed by Mathematical Reviews, Zentralblatt MATH, PASCAL CNRS Index, Referativnyi Zhurnal, Current Mathematical Publications and the Science Citation Index.

The Pacific Journal of Mathematics (ISSN 0030-8730) at the University of California, c/o Department of Mathematics, 969 Evans Hall, Berkeley, CA 94720-3840, is published monthly except July and August. Periodical rate postage paid at Berkeley, CA 94704, and additional mailing offices. POSTMASTER: send address changes to Pacific Journal of Mathematics, P.O. Box 4163, Berkeley, CA 94704-0163.

PJM peer review and production are managed by EditFLOW ${ }^{\mathrm{TM}}$ from Mathematical Sciences Publishers.

PUBLISHED BY PACIFIC JOURNAL OF MATHEMATICS

at the University of California, Berkeley 94720-3840

A NON-PROFIT CORPORATION

Typeset in IATEX

Copyright $(2011$ by Pacific Journal of Mathematics 


\title{
PACIFIC JOURNAL OF MATHEMATICS
}

\author{
Volume $252 \quad$ No. $2 \quad$ August 2011
}

Remarks on a Künneth formula for foliated de Rham cohomology

MÉLANIE BERTELSON

$K$-groups of the quantum homogeneous space ${ }_{q}(n) /_{q}(n-2)$

275

PARTHA SARATHI CHAKRABORTY and S. SUNDAR

A class of irreducible integrable modules for the extended baby TKK algebra

293

Xuewu Chang and ShaObin TAN

Duality properties for quantum groups

SOPHIE CHEMLA

Representations of the category of modules over pointed Hopf algebras over $\mathbb{S}_{3}$ and $\mathrm{S}_{4}$

Agustín García IGLESIAS and Martín Mombelli

$(p, p)$-Galois representations attached to automorphic forms on ${ }_{n}$

EKNATH GHATE and NARASIMHA KUMAR

On intrinsically knotted or completely 3-linked graphs 407

Ryo Hanaki, Ryo NikKUni, Kouki TaNiYama and AKIKo YAMAZAKI

Connection relations and expansions

Mourad E. H. ISMAIL and Mizan Rahman

Characterizing almost Prüfer $v$-multiplication domains in pullbacks

QING LI

Whitney umbrellas and swallowtails

TAKASHI NISHIMURA

The Koszul property as a topological invariant and measure of singularities

HAL SADOFSKY and BRAD SHELTON

A completely positive map associated with a positive map

ERLING STøRMER

Classification of embedded projective manifolds swept out by rational homogeneous varieties of codimension one

KiWAMU Watanabe

Note on the relations in the tautological ring of $\mu_{g}$ 